\title{
Forum
}

\section{The generalized replication principle and the partitioning of functional diversity into independent alpha and beta components}

\section{Zoltán Botta-Dukát}

Z. Botta-Dukát (http://orcid.org/0000-0002-9544-3474) (botta-dukat.zoltan@okologia.mta.hu), MTA Centre for Ecological Research, Vácrátót, Hungary.

\section{Ecography}

41: 40-50, 2018

doi: $10.1111 /$ ecog.02009

Subject Editor: Thiago Rangel. Editor-in-Chief: Pablo A. Marquet. Accepted 30 March 2017
The replication principle was first proposed by Hill (1973, Ecology 54: 247-432) as an advantageous property of his family of diversity indices. Later Jost (2007, Ecology 88: 2427-2439) discovered that diversity measures satisfying this principle allow partitioning of gamma diversity into independent alpha and beta components by simple multiplicative partitioning. Despite the emerging agreement on measuring taxonomic beta-diversity by multiplicative partitioning of Hill diversity, there is no consensus on how to measure functional beta diversity. Two different generalizations of Hill numbers for measuring functional diversity were proposed by Leinster and Cobbold (2011, Ecology 93: 477-489) and Chiu and Chao (2014, PLoS One 9: e1000014). Both generalizations attempted to satisfy the generalized replication principle, but they formulate it in different ways. The aims of this paper are 1) to review approaches for measuring functional diversity in units of equivalent numbers without explicit reference to replication principle; 2) to compare the two proposed replication principle and to point out some important differences in the behavior of diversity families derived from the two principles; 3 ) to explore the conditions necessary for partitioning functional diversity of Leinster and Cobbold into meaningful alpha and beta components; 4) and, finally, to explore how transformation of among-species distances into similarities influences the sensitivity of functional diversity to the scale parameter.

\section{Introduction}

Biodiversity is a complex and multifaceted concept that includes not only the number of species and their rarity (i.e. relative abundances) but also their functional (dis) similarity and evolutionary distinctness. While traditional diversity indices cannot capture functional or phylogenetic dissimilarities among species, many new indices have been proposed in the last decade that incorporate these aspects into diversity calculations (Pavoine and Bonsall 2011). Some of the new indices have been developed independently of the traditional diversity indices (Petchey and Gaston 2002, Villéger et al. 2008, Schmera et al. 2009); while others (Rao 1982, Ricotta and Szeidl 2006, Leinster and Cobbold 2011) can be regarded as extensions of traditional diversity indices and lead to the traditional formulas if the taxonomic distance

(C) 2017 The Author. Ecography (C) 2017 Nordic Society Oikos 
(i.e. the distance between every species pair is 1) is applied. The need for partitioning of phylogenetic or functional diversity into alpha and beta components naturally emerges for the latter group of indices because this partitioning may provide deeper insights into assembly processes (de Bello et al. 2009, Münkemüller et al. 2012).

The concept of alpha and beta diversity goes back to Whittaker's seminal paper (Whittaker 1960). However, for a long time there has been no consensus on how gamma diversity should be partitioned into two components (the proposed methods for calculating beta diversity were reviewed by Tuomisto 2010). Jost (2007) proved that multiplicative partitioning of Hill diversity leads to independent alpha and beta components. Formulas leading to independent alpha and beta components can be also derived for other diversity families (e.g. Rényi, Aczél-Daróczy, Patil-Tallie; see the review by Tóthmérész 1995 and the refererences therein) but they become more complicated (Jost 2007). The other virtue of Hill diversity is that it measures beta diversity in equivalent numbers (i.e. numbers of totally distinct subsamples that would lead to the same beta diversity value) (Jost 2007, Tuomisto 2010). Both of these desirable features of Hill diversity related to its so-called 'doubling property': if two equally sized subsamples without shared species have the same relative abundance distribution, than the Hill number of the pooled sample is doubled (Hill 1973). From Hill's proof it is clear that if $m$ equally sized subsamples (without shared species but with the same abundance distribution) are merged, the Hill diversity of the pooled sample is $m D$, where $D$ is the Hill diversity of one subsample. Recently, Chao et al. (2010) for their generalized Hill numbers, and independently Leinster and Cobbold (2011) for Hill's original formula proved a stronger version of this theorem: the local Hill-diversity in the two subsamples have to be equal, but the relative abundance distributions may differ. Based on this result the replication principle (Chakravarty and Eichhorn 1991) can be formulated in the following way: $\mathrm{D}$ is a true diversity measure only if the diversity of a pooled sample of $m$ maximally distinct and equally diverse subsamples is $\mathrm{m}$-times the diversity of a single subsample.

While applying Hill diversity and multiplicative partitioning is sometimes debated (Veech and Crist 2010), the consensus on their usefulness for calculating beta-diversity is emerging (Tuomisto 2010, Chao et al. 2012). Also there is an agreement in that the replication principle has to apply for functional and phylogenetic diversity indices if we want to partition them into alpha and beta components (Leinster and Cobbold 2011, Chiu and Chao 2014, Chiu et al. 2014), but the exact meaning of 'maximally distinct subsamples' and consequently the correct formulas are still debated. Leinster and Cobbold (2011) and Chiu and Chao (2014) proposed two different interpretations of the term 'maximally distinct' and therefore two different generalizations of Hill numbers for measuring functional diversity. Before comparing the two versions of replication principle and the derived generalizations of Hill numbers, I will shortly overview the approaches partitioning Rao's quadratic entropy (Rao 1982), the most widely used functional diversity index into independent alpha and beta components without referencing to replication principle. In the next section I show the two competing interpretations of the term 'maximally distinct subsamples' and derived formulas. Then, I prove that the generalized principle suggested by Chiu and Chao (2014) contradicts to the identical species principle proposed by Solow et al. (1993), and explore the conditions necessary for meaningful partitioning of the diversity measures proposed by Leinster and Cobbold (2011). In the last section I show that sensitivity of Leinster-Cobbold functional diversity to the scale parameter strongly depends on the transformation of distances between species into similarities between them.

\section{Pioneering approaches without referring to the replication principle}

Rao's quadratic entropy (Rao's Q) is the mean functional dissimilarity between two randomly chosen individuals. Since it can be easily interpreted and it satisfies the intuitive requirements for measuring functional alpha diversity (Botta-Dukát 2005), Rao's quadratic entropy became a popular measure of functional diversity. However, when it was tried to partition into alpha and beta components, problems arose; e.g. negative beta components (Villéger and Mouillot 2008) or unexpectedly low beta values, even for complete species replacement (de Bello et al. 2009). These problems led to a hot debate on the correct calculation of beta diversity (Ricotta 2005a, b, Hardy and Jost 2008, Villéger and Mouillot 2008, de Bello et al. 2010).

Neither of the approaches proposed in this debate referred to the (generalized) replication principle. In spite of this fact, results of de Bello et al. (2010) is worth a more detailed discussion, because they suggest a correct formula. They have started from the fact that Gini-Simpson diversity can be regarded as a special case of Rao's Q for taxonomic diversity, where $d_{i j}=1$ for every $i \neq j$ and $d_{i j}=0$ otherwise (BottaDukát 2005), and applied the transformation proposed by Jost (2007) for Gini-Simpson diversity:

${ }^{2} D=\frac{1}{1-Q}$

where ${ }^{2} D$ is the functional diversity measured in number of equivalents (value in superscript before $\mathrm{D}$ - here 2 - indicates the order of diversity, or, alternatively, the sensitivity parameter). This transformation was applied for both the Rao's Q calculated for the pooled sample (i.e. gamma diversity) and the arithmetic mean of Qs calculated for subsamples (i.e. alpha diversity), and then beta diversity was calculated as the ratio of the two transformed values:

$$
{ }^{2} D_{\beta}=\frac{1}{1-Q_{\gamma}} / \frac{1}{1-\sum_{i} Q_{i} / m}=\frac{1-\sum_{i} Q_{i} / m}{1-Q_{\gamma}}
$$

where $Q_{\gamma}=$ Rao's $Q$ in the pooled sample, $Q_{i}=$ Rao's $Q$ in the $\mathrm{i}$-th subsample, $m=$ number of subsamples. Note that 
this approach is valid only if the theoretical range of distance values is $[0,1]$, and thus the complement of distances can be interpreted as similarity. If this condition is not satisfied, we can divide all distance values by their theoretical maximum $\left(d_{\max }\right)$ and thus they will be scaled to be interval of $[0,1]$, or we can apply the same scaling for the calculated quadratic entropies:

$$
{ }^{2} D=\frac{1}{1-\sum_{i} \sum_{i}\left(d_{i j} / d_{\max }\right) p_{i} p_{j}}=\frac{1}{1-Q / d_{\max }}
$$

Ricotta and Acosta (2014) proposed using actual maximum of the distance values instead of the theoretical maximum that results in a formula applicable even if the distance function has no upper bound (e.g. Euclidean distance):

$$
{ }^{2} D^{*}=\frac{1}{1-Q / \max _{i, j} d_{i j}}
$$

Remind that by applying Eq. 4, the meaning of 'maximally distinct subsamples' depends on the dataset. Furthermore, functional diversity calculated by this formula does not obey the principle of monotonicity in distances (Weitzman 1992) since its value may decrease when some distances are increased while the others are remained constant (see an example in Table 1). Ricotta and Acosta (2014) also proposed using a pre-defined arbitrary value (denoted by $d^{\prime}$ ) instead of $d_{\text {max }}$. Since $d^{\prime}$ does not change automatically when distance values changes, this formula obeys the principle of monotonicity in distances. Note that for meaningful diversity values, $d$ ' has to be higher than $Q$. Thus including a new plot with high functional diversity may make necessary to choose a new, higher $d$ '. Since only diversity values calculated using the same $d$ ' are comparable, this does not lead to conflict with the principle of monotonicity in distances.

Chiu and Chao (2014) has pointed out another problem with applying Eq. 4. I agree with them that 'any sensible differentiation measure should not decrease', when 'the distance for any two species in different assemblages is either increased or kept as the same, whereas all the distances for species in the same assemblage are kept the same'. They exemplifies that transformation of Rao's Q by Eq. 4 does not always obey this criterion if the distance matrix is nonultrametric. Table 1 shows an example where non-sensible result appears, however the distance matrices are ultrametric.

\section{Two different generalizations of the replication principle}

Ricotta and Szeidl (2009) was the first who applied the replication principle for functional diversity. They generalized the principle as follows: 'if $\mathrm{N}$ equally diverse, equally large, and maximally dissimilar' subsamples 'are pooled, the diversity of the pooled' sample 'must be $\mathrm{N}$ times the diversity' of the subsamples. 'Maximally dissimilar' simply means no shared species if similarities among species are disregarded, but for calculating functional diversity it should be added that species in different subsamples are totally dissimilar. Using this generalized replication principle and definition of Rao's Q as mean difference between two randomly chosen individuals, they derived the transformation of Rao's Q into equivalent number of species. This derived formula is the same as proposed by de Bello et al. (2010).

Table 1. Comparison of two transformations (Eq. 1 and 4) of Rao's quadratic entropy (Q) into number of equivalents $\left({ }^{2} D\right.$ and $\left.{ }^{2} D^{*}\right)$.

\begin{tabular}{lccc}
\hline & $\begin{array}{c}\text { Case I } \\
\text { (ultrametric distance) }\end{array}$ & $\begin{array}{c}\text { Case III II } \\
\text { (non-ultrametric distance) }\end{array}$ & 0.05 \\
\hline $\mathrm{Q}_{\alpha}$ & 0.05 & 0.05 & 0.075 \\
$\mathrm{Q}_{\gamma} D_{\alpha}$ & 0.125 & 0.288 & 1.053 \\
${ }^{2} D_{\gamma}$ & 1.053 & 1.053 & 1.081 \\
${ }^{2} D_{\beta}$ & 1.143 & 1.404 & 1.027 \\
${ }^{2} D_{\alpha}^{* *}$ & 1.086 & 1.334 & 2 \\
${ }^{2} D_{\gamma}^{*}$ & 1.333 & 1.059 & 4 \\
${ }^{2} D_{\beta}^{*}$ & 2.666 & 1.471 & 2 \\
\hline
\end{tabular}

In each case there are two equal sized subsamples with the following relative abundance vectors: $P_{1}=[0.5,0.5,0,0]$ and $P_{2}=[0,0,0.5$,

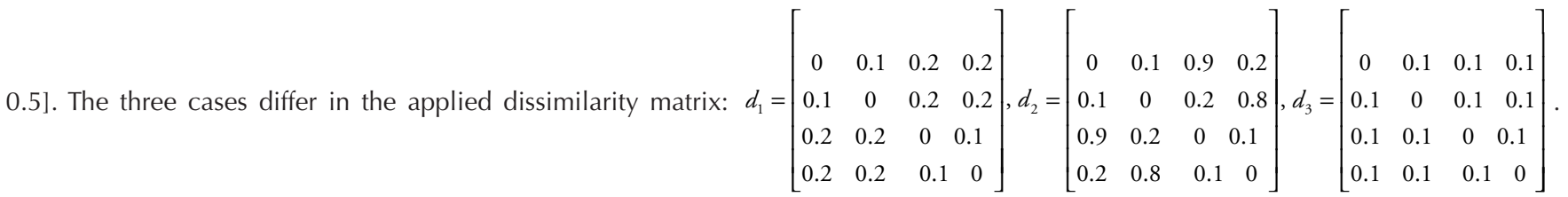

$d_{1}$ and $d_{3}$ are ultrametric distances matrices, while $d_{2}$ is non-ultrametric. Case I and II are adopted from Table 5 of Chiu and Chao (2014). Diversity values calculated using Eq. 1 (i.e. ${ }^{2} D$ ) behaved desirably: 1 ) alpha diversity is not influenced by changes distance between species pairs that never co-occur (i.e. it is the same in all cases); 2) gamma diversity decreases when differences either increased or kept constant (i.e. it is lower in Case II than in Case I); 3) beta diversity increases when the distance for any two species in different subsamples is either increased or kept as the same, whereas all the distances for species in the same subsample are kept the same.

Diversity values calculated using Eq. 4 (i.e. ${ }^{2} D^{*}$ ) show undesirable behavior: 1 ) alpha diversity is influenced by changes distance between species pairs that never co-occur; 2) gamma diversity increases when differences either increased or kept constant (i.e. it is lowest in Case III a highest in Case II); (3) beta diversity decreases (lower in Case II than is Case I) or remains constant (Case I and Case III) when the distance for any two species in different subsamples is either increased or kept as the same, whereas all the distances for species in the same subsample are kept the same. 
Leinster and Cobbold (2011; hereafter LC) and Chiu and Chao (2014; hereafter CC) proposed two different generalization of Hill diversity to incorporate differences between species (i.e. measuring functional diversity), and two corresponding generalization of the replication principle. Note that both formulas reduce to the original Hill diversity using specific distance matrix. A third formula for measuring functional diversity and inspired by Hill diversity was proposed by Scheiner (2012), but it does not refer to replication principle, therefore it will not be discussed in this paper.

LC's argumentation starts from the relationship between Hill diversity and generalized mean. The formula for $\mathrm{r}-\mathrm{th}$ order generalized mean of vector $\mathbf{x}$, with weights in vector $\mathbf{w}$ is:

$$
{ }^{r} M=\sum_{i=1}^{S}\left(w_{i} x_{i}^{r}\right)^{\frac{1}{r}}
$$

Generalized mean is a generalization of arithmetic mean, which is a special case for $r=1$. According to the definition by Patil and Taillie $(1979,1982)$ : diversity is the average rarity within a community weighted by relative abundances. Both Hill diversity and LC-diversity satisfy this definition if 'average' replaced by 'generalized mean of' (see proof in Supplementary material Appendix 1). For Hill diversity the rarity is measured by the reciprocal of relative abundances (Patil and Taillie 1982). LC pointed out that relative abundance is a measure of 'ordinariness' in the special case when similarity among species is zero, therefore they proposed mean similarity to species (including the focal species itself) as the general measure of 'ordinariness', and its reciprocal for measuring rarity.

LC generalized the replication principle similarly to Ricotta and Szeidl (2009): if a sample is divided into $m$ subsamples, no species appear in more than one subsample, species in different subsamples are totally dissimilar, and subsamples are equally sized and equally diverse, then diversity of the sample is $m D$, where $D$ is the diversity of a subsample. It follows the logic of the original replication principle, but inserts an additional condition (i.e. 'species in different subsamples are totally dissimilar'). Note that this condition would be superfluous for taxonomic diversity, where all species are regarded totally different.

CC first gave a simple derivation of Hill diversity: they suggest that $q$-th power sum of the relative abundances in the studied real sample and an idealized reference sample consisting of $D$ equally abundant species is equal, if $D$ is the $q$-th order diversity of the studied sample:

$$
\sum_{i=1}^{s} p_{i}^{q}=D\left(\frac{1}{D}\right)^{q} \Rightarrow D={ }^{q} D=\left(\sum_{i=1}^{s} p_{i}^{q}\right)^{1 /(1-q)}
$$

Then they applied this derivation to $p_{i} p_{j}$ products with weighting by the distance between $i$ and $j$ for deriving formulas for functional diversity:

$$
\begin{aligned}
\sum_{i=1}^{s} \sum_{j=1}^{s} d_{i j}\left(p_{i} p_{j}\right)^{q} & =D^{2} Q\left(\frac{1}{D} \frac{1}{D}\right)^{q} \Rightarrow D={ }^{q} D(Q) \\
& =\left(\sum_{i=1}^{s} \sum_{j=1}^{s} \frac{d_{i j}}{Q}\left(p_{i} p_{j}\right)^{q}\right)^{1 / 2(1-q)}
\end{aligned}
$$

Note that for taxonomic diversity the idealized reference sample consists of equally abundant species, while for functional diversity furthermore each element of the distance-matrix, including its diagonal elements (i.e. distance the species from itself) is $Q$.

The diversity index by CC obeys the original form of replication principle; i.e. no additional condition on dissimilarity among species from different subsamples has to be inserted.

Both the functional diversity indices suggested by $\mathrm{LC}$ and $\mathrm{CC}$ reduce to Hill numbers of taxonomic diversity but under different conditions. The index by LC equals to Hill diversity if the similarity between any species pair is zero, while similarity of each species to itself is one. (But note that if similarity between species is interpreted as expected similarity between two randomly selected individuals and within-species variation is considered, similarity of a species to itself may be lower than one.) This condition fits well to the intuition that in traditional taxonomic diversity each species pair is treated as maximally different. CC's functional diversity reduces to Hill's taxonomic diversity if each value of the distance matrix (including values in the main diagonal) is the same. The latter case can occur only if between-species distance is interpreted as expected difference between two individuals randomly selected from the compared species (otherwise there are zeros in the main diagonal of the distance matrix) and the expected difference between two randomly selected individuals is the same irrespectively of their taxonomic affiliation. In such situation intra- and interspecific variation of trait values is the same, therefore by my intuition functional diversity should be independent from taxonomic diversity, not equal to it.

Because of the difference in the interpretation of replication principle, the two functional diversity families differ in conditions necessary for maximizing beta-diversity. Functional beta diversity of $\mathrm{CC}$ is always maximal if the taxonomic beta-diversity is maximal (i.e. there are no shared species), irrespectively of the functional dissimilarities among species. It means that if there are no shared species, the original formulas become totally insensitive to the changes in trait values. However, they later developed modified versions of multiple dissimilarities (published as a comment to the original paper) that attain their maximum value if assemblages are completely distinct and any species-pair from different assemblages are maximally distinct. LC functional beta-diversity is maximal if each species occurs only in one subsample (i.e. there are no shared species and thus taxonomic beta diversity is maximal) and similarity between any two species occurring in different subsamples is zero.

Differences in the replication principle also leads to different units of functional diversity, LC functional diversity value of a community can be interpreted as number of equally abundant, functionally totally distinct species that would generate the same diversity. Thus it unit is number of species. The measure ${ }^{9} \mathrm{D}(\mathrm{Q})$ of $\mathrm{CC}$ (Eq. 7) "can be interpreted as 'the effective number of equally abundant and (functionally) equally distinct species' with a constant distance Q for all species pairs" (Chiu and Chao 2014), including distance of a species from itself. They also 
developed an other diversity measure ( ${ }^{9} \mathrm{FD}$ or total functional diversity) that measures the functional diversity in units of distance. It 'quantifies the effective total distance between species of the assemblage. If ${ }^{\mathrm{T}} \mathrm{FD}(\mathrm{Q})=\mathrm{u}$, then the effective total distance between species of the actual assemblage with quadratic entropy $Q$ is the same as that of an idealized assemblage having $(\mathrm{u} / \mathrm{Q})^{1 / 2}$ equally abundant and equally distinct species with a constant distance $\mathrm{Q}$ for all species pairs'.

\section{The functionally identical species principle}

This principle proposed by Solow et al. (1993): diversity should not be increased by the addition of a species if its minimum distance to the other occurring species is zero. More precise definition can be given by using the term 'functionally identical species' (Weitzman 1992). Two species $A_{1}$ and $A_{2}$ are functionally identical if the distance between them is zero $\left(d\left(A_{1}, A_{2}\right)=0\right)$ and they equally differ from any third species $\left(d\left(A_{1}, B\right)=d\left(A_{2}, B\right)\right)$. Merging functionally identical species into one species (e.g. A1 and A2 into A), or, in opposite, dividing a species aggregate into functionally identical but taxonomically distinct species should not change the functional diversity (Weitzman 1992). Taxonomically distinct but functionally identical species can be called twin species, therefore following Weitzman (1992) satisfying this principle could be referred to as 'twin property'.

The usefulness of this principle is debated. CC have added a comment to their paper where they argued that relevance of this principle depends on the goal of the study. They distinguished two major types of goals: relating functional diversity to ecosystem functioning (Tilman et al. 1997) and quantifying the community resilience or adaptive capacity in the face of environmental changes' (Walker et al. 1999). It does not influence the ecosystem functions that many functionally identical species play a role in the ecosystem; therefore twin property makes sense in the first approach. On the other hand, functionally redundant (twin) species may help to buffer the effect of species loss (Díaz and Cabido 2001); therefore the twin property is not necessary in the second approach. The third possible goal is exploring the rules of community assembly (Götzenberger et al. 2012). While the other two approaches are related to diversity of effect traits (Lavorel and Garnier 2002), in the community assembly studies the diversity of response traits are considered. Species with same response traits would behave as neutral species, whose abundance and distribution are driven only by stochasticity (Hubbell 2001). The aim of community assembly studies is detecting the departure from this random expectation (either convergence due to environmental filtering or divergence due to limiting similarity), thus twin property makes sense in this approach too.

LC functional diversity obeys the functionally identical species principle, while CC functional diversity does not. If it makes a problem, this issue can be solved within framework of CC (as they suggested in a comment to their paper) by replacing term 'species' with 'operational functional unit' (OFU) as proposed by Ricotta (2005a) and Schmera et al. (2009); two individuals belong to the same OFU, if they did not differ in their considered traits.

\section{Meaningful partitioning diversity into alpha and beta components}

The multiplicative diversity partitioning derived by Jost (2007) for equally weighted subsamples formally can be applied to LC functional diversity measure. While for taxonomic similarity matrix (i.e. Hill-diversity) beta-diversity always ranges between 1 and number of subsamples, applying Jost's approach for functional beta-diversity, it may lower than 1 , which is meaningless since the possible minimum of equivalent numbers is 1. CC's aim was to develop such a new version of generalized replication principle which guarantees that beta-diversity cannot be lower than 1 , thus it always interpreted as equivalent number. It has to be admitted that their measures satisfies this requirement, and in this respect it is better than LC diversity. However, I do not think that LC's functional diversity should be refused, rather we should look for the conditions that guarantee the meaningful partitioning instead.

Partitioning diversity into alpha and beta components is meaningful if gamma diversity $\left(D_{\gamma}\right)$ is higher than alpha diversity $\left(\mathrm{D}_{\alpha}\right)$. LC has shown that Ricotta-Szeidl's (2006) entropy $\left({ }^{\mathrm{Q}} \mathrm{H}\right)$ can be transformed into their diversity measure and vice versa. It is important since ${ }^{q} D_{\alpha} \leq{ }^{q} D_{\gamma}$ if the corresponding ${ }^{q} \mathrm{H}$ entropy is concave (see proof in the Supplementary material Appendix 1). For $q=2$ this entropy is the Rao's quadratic entropy which is concave if the matrix containing square-root of functional distances has Euclidean property (Champely and Chessel 2002). (Note that Ricotta (2005a) misstate - referring to the paper by Champely and Chessel - that the distance matrix itself should have this property.) In case studies, distance formulas derived from Gower's similarity are widely used. There are two ways to transform a similarity (s) into distance $(d): d=1-s$ or $d=\sqrt{1-s}$ (Podani 2000). The distance calculated by the later way has Euclidean property, if there is no missing data, but the this property is not assured in case of missing data (Pavoine et al. 2009). Thus Rao's quadratic entropy is concave, if 1 - Gower's similarity is used as distance, and there are no missing trait values. Species with missing values can be excluded from the calculation, especially if these species has low abundance. Note that papers often refer to Gower-distance without specifying the formula, which leads to ambiguity. 'gowdis' function in the FD package (Laliberté et al. 2014) uses the first transformation.

Another possible measure of the functional dissimilarity between species is the complement of the overlap between their trait distribution (de Bello et al. 2013, Carmona et al. 2016). It is a special case of Bray-Curtis distance, whose square-root has Euclidean property (Legendre and De Cáceres 2013).

Ricotta and Szeidl (2006) proved that ${ }^{q} \mathrm{H}$ is concave for $q \geq 2$ if the similarity matrix of species is positive semi-definite 
(i.e. neither of its eigenvalue is negative). Gower and Legendre (1986) has proved that a similarity matrix is positive semi-definite if matrix of distances in $d=\sqrt{1-s}$ form has Euclidean property. Thus theorem of Ricotta and Szeidl (2006) is a generalization of Champely and Chessel's above mentioned theorem on concavity of Rao's Q. Unfortunately, they cannot find any general rule for $q<2$ that guarantees holding concavity. Note that Euclidean property of a distance formula guarantees that distance matrices calculated by this way are always Euclidean, but distance matrices may have this property, even if they were calculated by a 'non-Euclidean' formula. Furthermore, functional diversity may be concave even if the similarity matrix is not positive semi-definite, however only positive semi-definiteness and scale parameter $q \geq 2$ guarantee the concavity. Therefore, I recommend preferring similarity measures that guarantees positive semi-definiteness, only if they behave well otherwise (for comparing behavior of similarity measures see the next section). On the other hand, if someone choose parameter setting that does not guarantees concavity $(q<2$ or the similarity matrix is not positive semidefinite), she/he should carefully check the beta-diversity values and should change the settings, if concavity is not satisfied (i.e. lower than one beta diversity values appear).

\section{Sensitivity to the value of scale parameter}

In Hill diversity, scale parameter sets the sensitivity of the index to the rare species: lower value leads to higher sensitivity for species with low abundances. Two extreme values are zero, where Hill diversity is number of species disregarding their relative abundances, and infinity, where the diversity is the reciprocal of the highest relative abundance, thus it depends on the relative abundance of the most abundant species only. Scale parameter in LC diversity also sets the weighting of rare species, but here rarity of species depends on the relative abundance of the similar species (weighted by their similarity) beyond the species own abundance. CC criticized LCdiversity on the ground that it 'may not be sensitive to species abundances when species similarity matrix is computed from species traits in functional analysis'. In this section I argue that it is not an inherent property of LC-diversity, rather a byproduct of the widely used transformation of distances into similarities.

Remember, LC-diversity is based on functional similarities, not functional distances. Functional similarities range from 0 (totally different species) to 1 (functionally identical species). If functional distance also ranges in this interval (e.g. Gover distance), a straightforward way of transformation is:

$s_{i j}=1-d_{i j}$

If distance may be higher than 1 , it should be re-scaled first into this interval. The problems of re-scaling using the actual maximum has already been discussed above. Hereafter, I will refer to this transformation as linear transformation.
LC proposed another transformation (hereafter called exponential) transformation:

$s_{i j}=\exp \left(-u * d_{i j}\right)$

where $u(>0)$ is a scaling constant that influence the shape of the relationship between distances and similarities. For higher $u$, similarity decrease more steeply with increasing distance. The merit of exponential transformation is that it results similarity between zero and one, irrespectively the original range of the distance. On the other hand, similarity cannot reach the zero (i.e. totally different species), if the distance value is finite.

To overcome this drawback of exponential transformation, I propose the following modified exponential transformation:

$s_{i j}= \begin{cases}\frac{\exp \left(-u * d_{i j}\right)-\exp (-1)}{1-\exp (-1)} & \text { if } d_{i j}<1 / u \\ 0 & \text { otherwise }\end{cases}$

Using this transformation, similarity decrease first exponentially with increasing distance, but above the threshold $(1 / u)$ it is zero, irrespectively the distance.

Further transformations could be developed (e.g. LC proposed piece-wise linear transformation), with the restriction similarity has to be 1 , if the distance is zero.

To compare the behavior of LC-diversity with different similarity matrices, I calculated the functional diversity for 103 plots of Lhotsky et al. (2016a). Plots were located along a productivity gradient from open sand grasslands through meadows to wetlands, thus there are large differences in traits of the occurring 239 species. In this analysis only the four continuous traits (clonal height, leaf size, SLA, and LDMC) were used, where data on intraspecific variation was available for about half of the species from our own measurements (Lhotsky et al. 2016b). Five similarity matrices were compared: 1 - taxonomic dissimilarity (the identity matrix), linear transformation of Gower-dissimilarity, exponential transformation of Gower-dissimilarity with $u=10$, modified exponential transformation of Gower-dissimilarity with $u=10$, arithmetic and geometric means of overlaps calculated for each four traits separately. Overlap of trait distribution were calculated for each trait separately by supposing Gaussian distribution (approach 1 in de Bello et al. 2013) and then arithmetic (Lepš et al. 2006) or geometric mean of overlap values are calculated. Since I have no data on intraspecific trait variation for all species, the same common standard deviation value estimated from the available data were used.

Ranking of the plots according to their functional (alpha) diversity is hardly changed, if different similarity matrix were used (Supplementary material Appendix 1-2). However, sensitivity functional alpha diversity to scale parameter (q) depends on the applied similarity matrix: if linear transformation of Gower-distance was used, functional diversity proved to be insensitive to the scale parameter, since values are near 
to the possible minimum (Fig. 1a). Exponential transformation of Gower-distance and arithmetic mean of overlaps show similar pattern, but they were slightly more sensitive (Fig. 1b, d). Contrary to them, modified exponential transformation of Gower-distance and geometric mean of overlaps
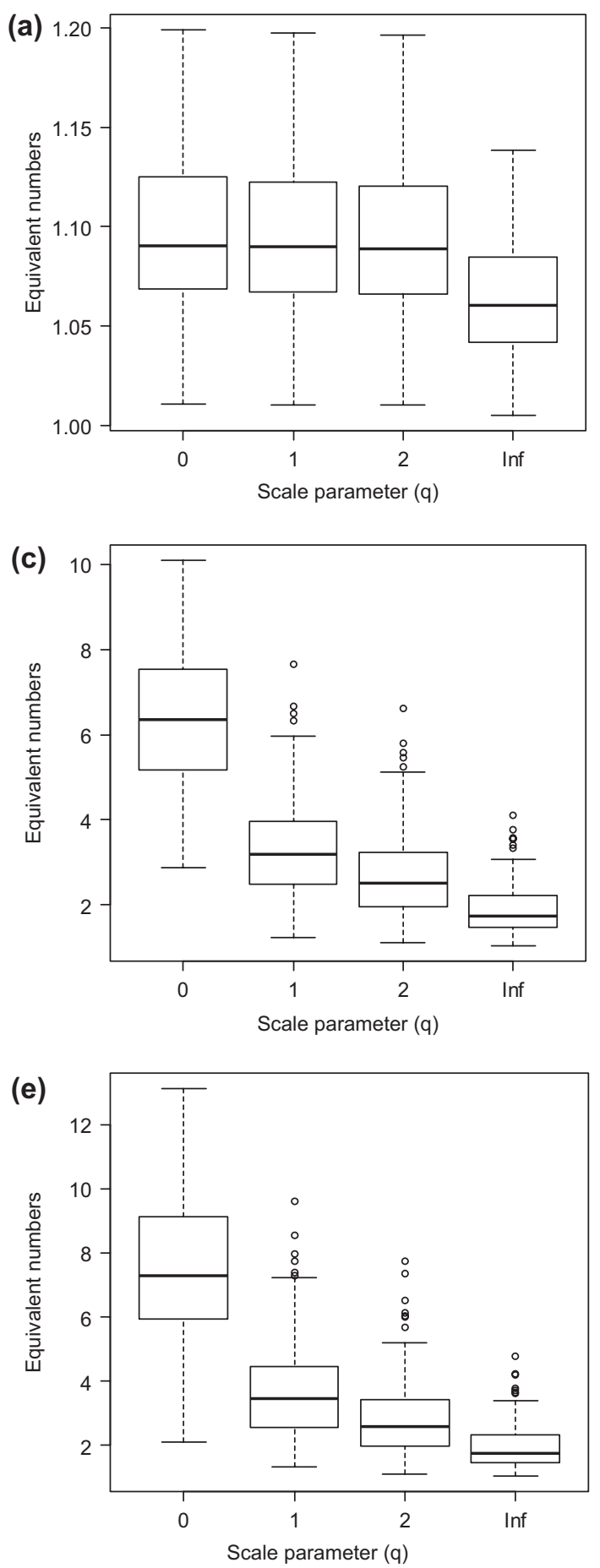

lead to functional diversity that sensitive to scale parameter (Fig. 1c, e).

CC gave a clue for the explanation of this pattern. They wrote: 'if species similarity matrix deviates greatly from a naïve identity matrix, then their measure typically yields
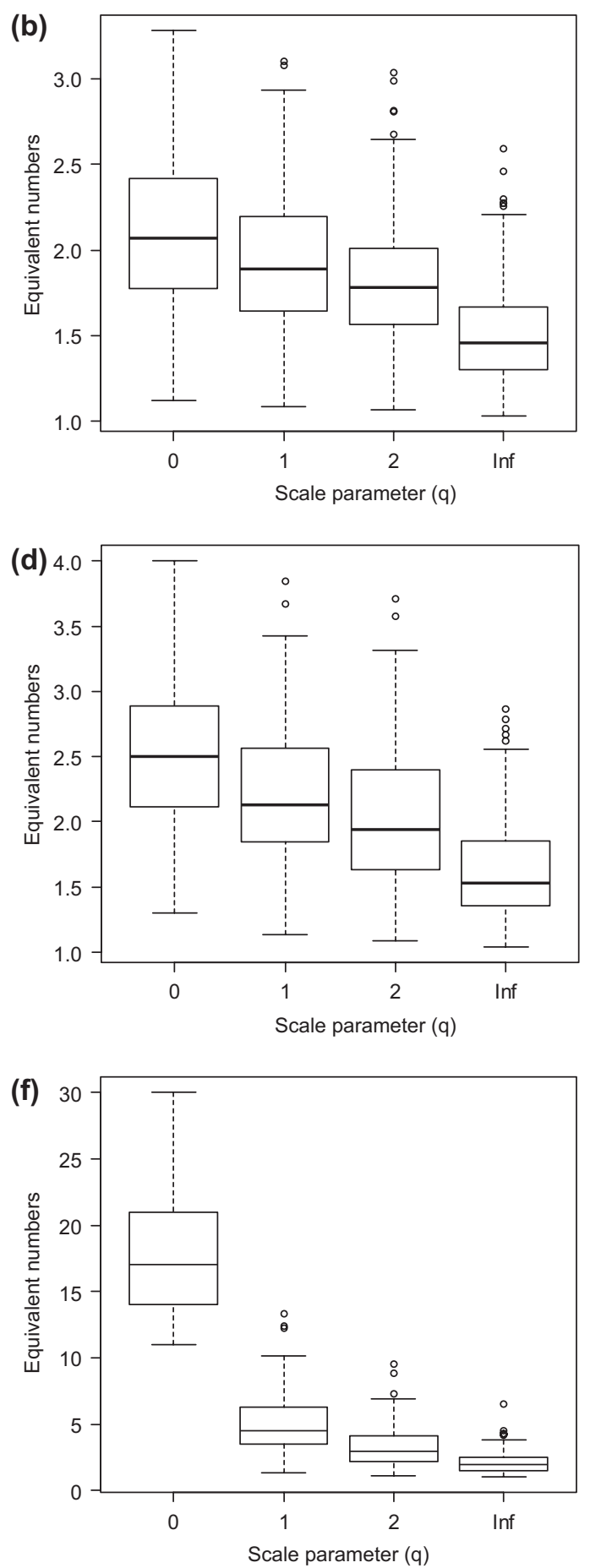

Figure 1. Functional (a-e) and taxonomic (f) alpha diversity in 103 plots of the dataset by Lhotsky et al. (2016a) at different scale parameter $(q)$ values. Functional diversity were calculated by LC diversity, using different similarity matices: (a) linear transformation of Gower-distance, (b) exponential transformation Gower-distance with $\mathrm{u}=10$, (c) modified exponential transformation Gower-distance with $\mathrm{u}=10$, (d) arithmetic mean of overlaps, (e) geometric mean of overlaps. 
very low diversity values'. LC-diversity is equivalent to taxonomic diversity, if all non-diagonal element of the similarity matrix is zero, i.e. species are totally distinct. It is the possible maximum of LC-diversity. LC-diversity reaches its minimum, when all non-diagonal elements are 1, i.e. all species are functionally equivalent. LC has shown that increasing

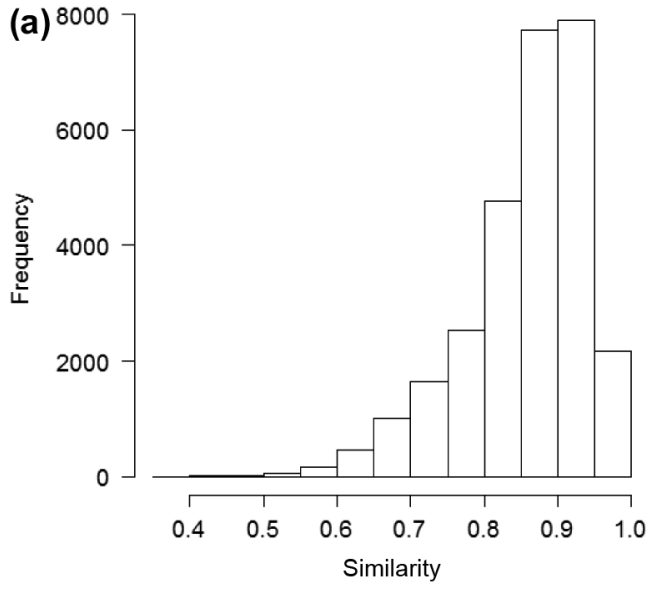

(c)

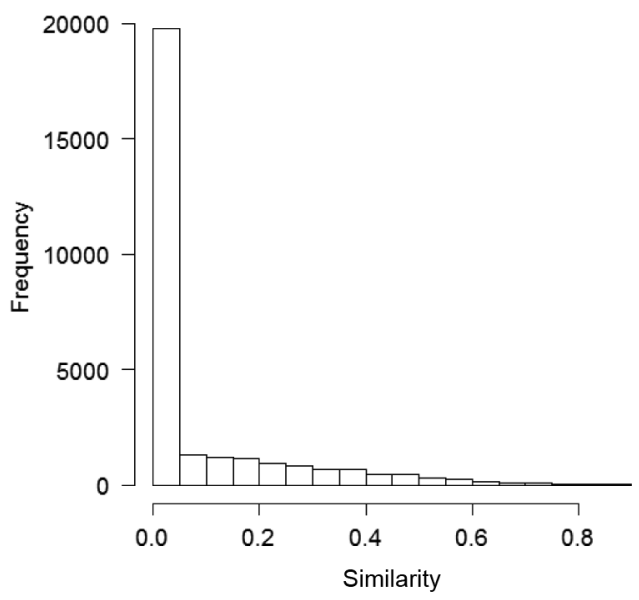

the similarities decrease the functional diversity. Since the possible minimum is independent from the scale parameter, if values decrease to near the minimum, diversity becomes insensitive to the scale parameter.

Figure 2 shows the histogram of similarities (excluding the similarity of species to themselves). If linear transformation
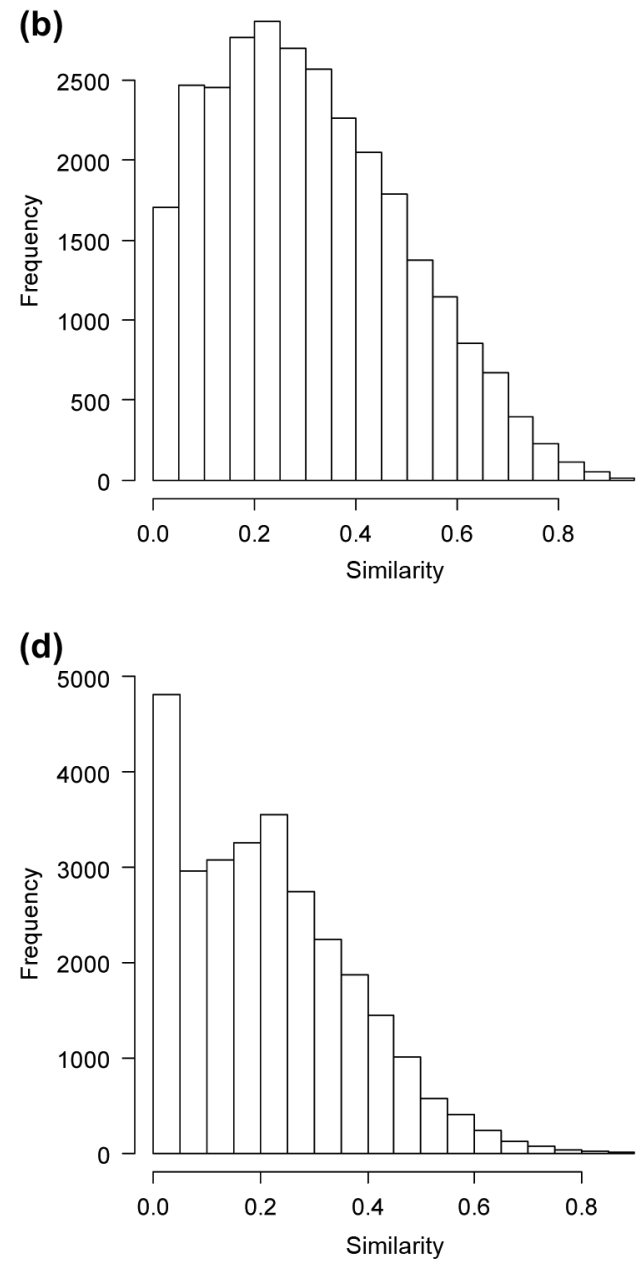

(e)

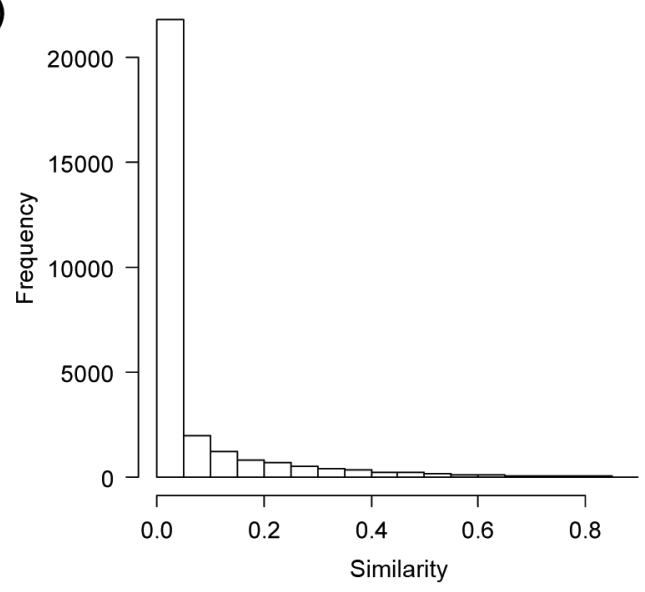

Figure 2. Histogram of the off-diagonal elements of the similarity matrices used for calculation of functional diversity in Fig. 1. (a) Linear transformation of Gower-distance, (b) exponential transformation Gower-distance with $\mathrm{u}=10$, (c) modified exponential transformation Gower-distance with $\mathrm{u}=10$, (d) arithmetic mean of overlaps, (e) geometric mean of overlaps. 
of Gower-distance is applied, most of the similarity is high, indicating that species are regarded to be almost functionally equivalent. This strange distribution of similarities is not specific to this dataset, Fig. 2 in de Bello et al. (2013) shows similar histogram (note that they drew histogram dissimilarities $=1$-similarities). It comes from the fact that Gower distance standardizes the variables by their range. Consequently, distance may be low for functionally rather different species, especially when the between-species variation is high (i.e. the range of trait values is wide). If exponential transformation is applied, decrease of similarity with increasing distance is steeper. Therefore the distribution of similarities is unimodal (Fig. 2b); both the largest and lowest values are rare. It less contradicts our intuition, but still considerably differs from the pattern of taxonomic similarity, where all values are zero. Only modified exponential transformation leads to high number of zero similarities (i.e. high number of totally functionally distinct species pairs), therefore only this transformation of Gower-distance leads to functional diversity sensitive to scale parameters. Arithmetic mean of overlaps may be medium, if variation ranges of some traits considerably overlap, while the overlap is zero in other traits. Whereas geometric mean is zero if there is no overlap in any trait.

In my opinion, if we think that distribution of similarities should look like Fig. 2a (i.e. calculated by linear transformation of Gower-distance), we have to accept that LC functional alpha diversity will be insensitive to changes in scale parameter. On the other hand, if we expect LC functional alpha diversity to be sensitive to scale parameter, we have to choose similarity measure that leads to distribution looks like Fig. 2c. In our example modified exponential transformation of Gower-distance and geometric mean of overlaps results in such distribution.

Functional beta diversity of the 103 plots proved to be insensitive to scale parameter irrespectively to the applied similarity matrix (Fig. 3). Whereas beta diversity values strongly depends on the applied similarity. We cannot know the correct value, but values near to one (i.e. that we have got using linear transformation of Gower dissimilarity) are clearly too low for such a long environmental gradient.

Before choosing the similarity measure one should answer two questions. The first question is that is the sensitivity to scale parameter an expected property of the functional (alpha) diversity in that study?' In my opinion, the answer to this question is often 'no'. Taxonomic diversity calculated for low and high scale parameter may rank sets of communities in different ways (Tóthmérész 1995). This pattern helps to better understand the contribution of dominant and rare species to diversity. Keep in mind that Hill-diversity is the weighted generalized mean of rarity, where scale parameter sets the emphasis on low and high rarity values. Since high rarity means low relative abundance, the interpretation of diversity profiles is straightforward. LC functional diversity is the reciprocal of the weighted generalized mean of ordinariness (Leinster and Cobbold 2011). Even a rare species may have high ordinariness due to high sum-

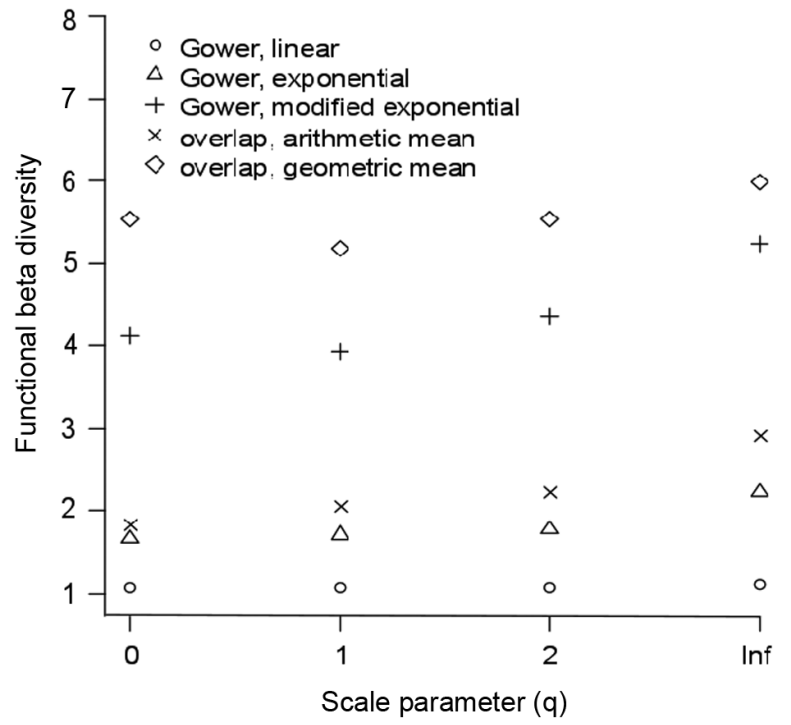

Figure 3. Beta diversity calculated for the whole dataset (i.e. 103 plots) using different similarity matrices and scale parameter $(q)$ values.

marized abundance of the similar species. The interpretation of ordinariness is less straightforward than interpretation of rarity. Probably it is the reason of rare application of diversity ordering in functional diversity studies. Sensitivity to scale parameter is a desirable property of the functional diversity, only if one thinks that giving different emphasis on low and high ordinariness values can help in better understanding the studied object.

The second question is that 'is there any other objective way to choose the most appropriate similarity measure?' The answer is often 'yes', however there is no general algorithm for selecting the most appropriate similarity function. It depends on the available data and purpose of the study. For example, if there are no data on within species variability, overlap of trait distribution cannot be chosen. If such data are available, one can choose overlap on theoretical ground (see Carmona et al. 2016 and references therein for detailed reasoning). Overlap is calculated for each trait separately and then averaged. Depending on the purpose one can prefer arithmetic or geometric mean. Arithmetic mean is suitable if traits are related to independent functions, and thus a species can substitute an other species in some functions even if their distributions do not overlap in some traits at all. Geometric mean should be used, when species can substitute each other, only if they are similar in all traits. Correlation with environmental data also can be involved the selection process. For example, it can be hypothesized that there is a positive correlation between difference in environmental conditions and difference in functional trait composition (i.e. plots from similar environment are similar in trait compostion, while plots from markedly different environments are also different in trait composition). Difference in trait composition can be measured by functional beta-diversity calculated for plot-pairs, and then the Mantel-correlation between matrix of these beta-diversities and distances in the space of environmental variables can be calculated. In 
Table 2. Comparing some properties of the two functional diversity families developed from different generalization of replication principle. Desirable properties are highlighted by bold typeface.

\begin{tabular}{|c|c|c|}
\hline Property & LC functional diversity & CC functional diversity \\
\hline Obeys replication principle & $\begin{array}{l}\text { in its modified version (see details in } \\
\text { the main text) }\end{array}$ & in its original form \\
\hline $\begin{array}{l}\text { Reduces to original Hill diversity, } \\
\text { if... }\end{array}$ & $\ldots d_{i j}=1$ for every $i \neq j$ & $\ldots$ if $d_{i j}=$ constant, for every $i$ and $j$, including $i=j$ \\
\hline $\begin{array}{l}\text { If there are no shared species, } \\
\text { functional beta-diversity... }\end{array}$ & $\begin{array}{l}\text {... depends on the trait similarities } \\
\text { among subsamples }\end{array}$ & $\begin{array}{l}\text {... always maximal, but multiple dissimilarity measures } \\
\text { sensitive to trait similarities are proposed }\end{array}$ \\
\hline Obeys 'twin property': & Yes & $\begin{array}{l}\text { only if species merged into operational functional units } \\
\text { before the analysis }\end{array}$ \\
\hline $\begin{array}{l}\text { Sensitive to the values of scale } \\
\text { parameter }\end{array}$ & $\begin{array}{l}\text { depends on the way to transform } \\
\text { distances into similarities }\end{array}$ & always \\
\hline
\end{tabular}

our Orgovány dataset NDVI were the only available environmental variable that used as proxy of the habitat productivity. Table 3 shows the values of Mantel-correlation for different scaling parameter (q) values and different similarity matrices (including the taxonomic beta-diversity as a special case). In this example, functional beta diversity calculated with linear and exponential transformation of Gowerdissimilarity has lower correlation values than the taxonomic beta diversity, thus based on this test they should not selected. Modified exponential transformation of Gower dissimilarity and arithmetic mean of overlaps performs slightly better than taxonomic similarity, but based on this criterion the best choice is the geometric mean of overlaps. Note that it is only an illustrative example whose results cannot be generalized.

In summary, insensitivity to scale parameters is not an inherent property of LC-diversity, but a by-product of Gower-distance combined with linear transformation. To avoid the insensitivity, similarity matrix with lot of low off-diagonal values should be used in the calculation of functional diversity. This property of the similarity matrix can (and should) be checked for the actual dataset by plotting the histogram of similarities, before calculation of functional diversity.

\section{Conclusion}

The aim of this paper was to compare two families of functional diversity indices: CC family of indices that obey the original version of replication principle, but (without merging functionally identical species) does not obey the functionally identical species principle, and LC family of indices that based on a generalized replication principle. Properties of the two families of functional diversity are summarized in Table 2. It can be seen that both generalization has its own virtues. The importance of these virtues strongly depends on the aim of the study. For example, obeying identical species principle is a necessary condition of a functional diversity measure used for relating functional diversity to ecosystem functioning or exploring rules of community assembly. Therefore, I recommend using LC measure for these purposes. After merging functionally identical species into operational functional units CC measure also can be used in diversity-ecosystem function studies, but this solution cannot be used in community assembly studies. On the other hand, twin property is not necessary when our aim is quantifying the community resilience. In such cases CC measure probably outperforms LC functional diversity.

Acknowledgement - Thanks to Francesco de Bello, Imelda Somodi and Attila Lengyel for their helpful comments. The work was supported by NKFIH (project nr. OTKA K83595).

\section{References}

Botta-Dukát, Z. 2005. Rao's quadratic entropy as a measure of functional diversity based on multiple traits. - J. Veg. Sci. 16: $533-540$.

Table 3. Mantel-correlation between difference in NDVI (as proxy of productivity) and pairwise LC beta diversity calculated using different similarity matrices.

\begin{tabular}{|c|c|c|c|c|}
\hline & \multicolumn{4}{|c|}{ Scale parameter $(q)$} \\
\hline & 0 & 1 & 2 & Inf \\
\hline Taxonomic similarity & $0.4690^{* * * *}$ & $0.1299^{\mathrm{NS}}$ & $0.0227^{\mathrm{NS}}$ & $-0.0430^{\mathrm{NS}}$ \\
\hline Gower, exponential, $\mathrm{u}=10$ & $0.3393^{* * *}$ & $0.2849^{* * *}$ & $0.2392^{* *}$ & $0.1581^{\mathrm{NS}}$ \\
\hline Gower, modified Exponential, $u=10$ & $0.5068^{* * *}$ & $0.2709^{* * *}$ & $0.1706^{*}$ & $0.1075^{\mathrm{NS}}$ \\
\hline Overlap, arithmetic mean & $0.4936^{* * *}$ & $0.3679^{* * * *}$ & $0.2853^{* * *}$ & $0.1782 *$ \\
\hline Overlap, geometric mean & $0.7284^{* * * *}$ & $0.3719^{* * * *}$ & $0.2298^{* *}$ & $0.1424^{\mathrm{NS}}$ \\
\hline
\end{tabular}


Carmona, C. P. et al. 2016. Traits without borders: integrating functional diversity across scales. - Trends Ecol. Evol. 31: 382-394.

Chakravarty, S. and Eichhorn, W. 1991. An axiomatic characterization of a generalized index of concentration. - J. Product. Anal. 2: 103-112.

Champely, S. and Chessel, D. 2002. Measuring biological diversity using Euclidean metrics. - Environ. Ecol. Stat. 9: 167-177.

Chao, A. et al. 2010. Phylogenetic diversity measures based on Hill numbers. - Phil. Trans. R. Soc. B 365: 3599-3609.

Chao, A. et al. 2012. Proposing a resolution to debates on diversity partitioning. - Ecology 93: 2037-2051.

Chiu, C.-H. and Chao, A. 2014. Distance-based functional diversity measures and their decomposition: a framework based on Hill numbers. - PLoS One 9: e100014.

Chiu, C.-H. et al. 2014. Phylogenetic beta diversity, similarity, and differentiation measures based on Hill numbers. - Ecol. Monogr. 84: 21-44.

de Bello, F. et al. 2009. Partitioning of functional diversity reveals the scale and extent of trait convergence and divergence. - J. Veg. Sci. 20: 475-486.

de Bello, F. et al. 2010. The partitioning of diversity: showing Theseus a way out of the labyrinth. - J. Veg. Sci. 21: 992-1000.

de Bello, F. et al. 2013. Which trait dissimilarity for functional diversity: trait means or trait overlap? - J. Veg. Sci. 24: 807-819.

Díaz, S. and Cabido, M. 2001. Vive la différence: plant functional diversity matters to ecosystem processes. - Trends Ecol. Evol. 16: 646-655.

Götzenberger, L. et al. 2012. Ecological assembly rules in plant communities - approaches, patterns and prospects. - Biol. Rev. 87: 111-127.

Gower, J. C. and Legendre, P. 1986. Metric and Euclidean properties of dissimilarity coefficients. - J. Classif. 3: 5-48.

Hardy, O. J. and Jost, L. 2008. Interpreting and estimating measures of community phylogenetic structuring. - J. Ecol. 96: 849-852.

Hill, M. O. 1973. Diversity and evenness: a unifying notation and its consequences. - Ecology 54: 427-432.

Hubbell, S. P. 2001. A unified theory of biodiversity and biogeography. - Princeton Univ. Press.

Jost, L. 2007. Partitioning diversity into independent alpha and beta components. - Ecology 88: 2427-2439.

Laliberté, E. et al. 2014. FD: measuring functional diversity from multiple traits, and other tools for functional ecology. - R package ver. $1.0-12$.

Lavorel, S. and Garnier, E. 2002. Predicting changes in community composition and ecosystem functioning from plant traits: revisiting the Holy Grail. - Funct. Ecol. 16: 545-556.

Legendre, P. and De Cáceres, M. 2013. Beta diversity as the variance of community data: dissimilarity coefficients and partitioning. - Ecol. Lett. 16: 951-963.

Leinster, T. and Cobbold, C. A. 2011. Measuring diversity: the importance of species similarity. - Ecology 93: 477-489.

Lepš, J. et al. 2006. Quantifying and interpreting functional diversity of natural communities: practical considerations matter. - Preslia 78: 481-501.

Lhotsky, B. et al. 2016a. Changes in assembly rules along a stress gradient from open dry grasslands to wetlands. - J. Ecol. 104: 507-517.

Lhotsky, B. et al. 2016b. New plant trait records of the Hungarian flora. - Acta Bot. Hung. 58: 397-400.

Supplementary material (Appendix ECOG-02009 at < www. ecography.org/appendix/ecog-02009>). Appendix 1-2.
Münkemüller, T. et al. 2012. From diversity indices to community assembly processes: a test with simulated data. - Ecography 35: 468-480.

Patil, G. P. and Taillie, C. 1979. An overview of diversity. - In: Grassle, J. F. et al. (eds), Ecological diversity in theory and practice. International Cooperative Publishing House, pp. 3-27.

Patil, G. P. and Taillie, C. 1982. Diversity as a concept and its measurement. - J. Am. Stat. Assoc. 77: 548-561.

Pavoine, S. and Bonsall, M. B. 2011. Measuring biodiversity to explain community assembly: a unified approach. - Biol. Rev. 86: 792-812.

Pavoine, S. et al. 2009. On the challenge of treating various types of variables: application for improving the measurement of functional diversity. - Oikos 118: 391-402.

Petchey, O. L. and Gaston, K. J. 2002. Functional diversity (FD), species richness and community composition. - Ecol. Lett. 5: 402-411.

Podani, J. 2000. Introduction to the exploration of multivariate biological data. - Backhuys Publishers.

Rao, C. R. 1982. Diversity and dissimilarity coefficients: a unified approach. - Theor. Popul. Biol. 21: 24-43.

Ricotta, C. 2005a. A note on functional diversity measures. - Basic Appl. Ecol. 6: 479-486.

Ricotta, C. 2005b. Additive partitioning of Rao's quadratic diversity: a hierarchical approach. - Ecol. Model. 183: 365-371.

Ricotta, C. and Szeidl, L. 2006. Towards a unifying approach to diversity measures: bridging the gap between the Shannon entropy and Rao's quadratic index. - Theor. Popul. Biol. 70: 237-243.

Ricotta, C. and Szeidl, L. 2009. Diversity partitioning of Rao's quadratic entropy. - Theor. Popul. Biol. 76: 299-302.

Ricotta, C. and Acosta, A. 2014. On the functional diversity of partially distinct species: some theory and a practical example. - Commun. Ecol. 15: 205-211.

Scheiner, S. M. 2012. A metric of biodiversity that integrates abundance, phylogeny, and function. - Oikos 121: 1191-1202.

Schmera, D. et al. 2009. A measure for assessing functional diversity in ecological communities. - Aquat. Ecol. 43: 157-167.

Solow, A. et al. 1993. On the measurement of biological diversity. - J. Environ. Econ. Manage. 24: 60-68.

Tilman, D. et al. 1997. the influence of functional diversity and composition on ecosystem processes. - Science 277: 1300-1302.

Tóthmérész, B. 1995. Comparison of different methods for diversity ordering. - J. Veg. Sci. 6: 283-290.

Tuomisto, H. 2010. A diversity of beta diversities: straightening up a concept gone awry. Part 1. Defining beta diversity as a function of alpha and gamma diversity. - Ecography 33: 2-22.

Veech, J. A. and Crist, T. O. 2010. Diversity partitioning without statistical independence of alpha and beta. - Ecology 91: 1964-1969.

Villéger, S. and Mouillot, D. 2008. Additive partitioning of diversity including species differences: a comment on Hardy \& Senterre (2007). - J. Ecol. 96: 845-848.

Villéger, S. et al. 2008. New multidimensional functional diversity indices for a multifaceted framework in functional ecology. - Ecology 89: 2290-2301.

Walker, B. et al. 1999. Plant attribute diversity, resilience, and ecosystem function: the nature and significance of dominant and minor species. - Ecosystems 2: 95-113.

Weitzman, M. L. 1992. On diversity. - Q. J. Econ. 107: 363-405.

Whittaker, R. H. 1960. Vegetation of the Siskiyou Mountains, Oregon and California. - Ecol. Monogr. 30: 279-338. 